\title{
Manipulation via Endowments in Exchange Markets with Indivisible Goods*
}

\author{
Murat Atlamaz ${ }^{\dagger} \quad$ Bettina Klaus ${ }^{\ddagger}$
}

September 2005

\begin{abstract}
We consider exchange markets with heterogeneous indivisible goods. We are interested in exchange rules that are efficient and immune to manipulations via endowments (either with respect to hiding or destroying part of the endowment or transferring part of the endowment to another trader). We consider three manipulability axioms: hiding-proofness, destruction-proofness, and transfer-proofness. We prove that no rule satisfying efficiency and hiding-proofness (which together imply individual rationality) exists. For two agents with separable and responsive preferences, we show that efficient, individually rational, and destruction-proof rules exist. However, for some profiles of separable preferences, no rule is efficient, individually rational, and destruction-proof. In the case of transfer-proofness the compatibility with efficiency and individual rationality for the two-agent case extends to the unrestricted domain. If there are more than two agents, for some profiles of separable preferences, no rule is efficient, individually rational, and transfer-proof.
\end{abstract}

JEL Classification: C71, D63, D71.

Keywords: Hiding-proofness, destruction-proofness, transfer-proofness, exchange markets with heterogeneous indivisible objects.

\section{Introduction}

We consider exchange markets with heterogeneous indivisible objects where each agent is endowed with a set of objects. As an example, one may think of markets where people trade collectibles, for instance stamps, Pockeymon cards, etc.. Other applications (see also Pápai, 2003) are exchanges of equipment or tasks among workers or departments of a firm or an organization. A well-known special case of our exchange model are so-called housing markets (Shapley and Scarf, 1974) where each agent is endowed with exactly one object. For housing markets, the so-called top trading rule that assigns the unique core allocation to each housing market satisfies many appealing properties. In particular,

\footnotetext{
${ }^{*}$ We thank Somdeb Lahiri, William Thomson, and an anonymous referee for helpful comments.

${ }^{\dagger}$ Department of Economics, University of Rochester, Rochester, NY 14627, USA; e-mail: muat@troi.cc.rochester.edu.

${ }^{\ddagger}$ Corresponding Author: Department of Economics, Maastricht University, P.O. Box 616, 6200 MD Maastricht, The Netherlands; e-mail: B.Klaus@algec.unimaas.nl; tel.: +31-433884605; fax: +31433884878. B. Klaus's research was partly supported by a Ramón y Cajal contract and Research Grant BEC2002-02130 from the Spanish Ministerio de Ciencia y Tecnología and by the Barcelona Economics Program of CREA.
} 
the top trading rule is efficient and strategy-proof [no agent ever benefits from misrepresenting her preferences] (Roth, 1982). Moreover, it is the only rule satisfying efficiency, strategy-proofness, and individual rationality [no agent is worse off after trading with other agents] (Ma, 1994). However, this compatibility result does not extend to "multiple object" exchange markets (Sönmez, 1999; Klaus and Miyagawa, 2002). Some recent studies for exchange markets with indivisibilities and multiple assignment problems without endowments that consider strategy-proofness in combination with other properties are Ehlers and Klaus (2003), Klaus and Miyagawa (2002), and Pápai (2003, 2004).

We are interested in efficient and individually rational exchange rules. In addition, we do not want any trader to be able to successfully manipulate the outcome to her advantage by hiding or destroying part of her endowment or transferring part of it to another trader who is not worse off because of the transfer. ${ }^{1}$ We call an exchange rule that is immune to this type of manipulation hiding-proof, destruction-proof, and transfer-proof, respectively.

In the context of classical exchange economies, Postlewaite (1979) is the first to introduce and study hiding-proofness and destruction-proofness. He shows that, when preferences are continuous, strictly increasing, and strictly convex, hiding-proofness is incompatible with efficiency and individual rationality. He also shows that destructionproofness is compatible with efficiency and individual rationality. ${ }^{2}$ For reallocation problems with single-peaked preferences, Klaus, Peters, and Storcken (1997) consider hidingproof rules satisfying various fairness and/or consistency properties. In the context of twosided matching with endowments, Sertel and Özkal-Sanver (2002) and Fiestras-Janeiro, Klijn, and Sánchez (2004) analyze the manipulability of men- (women-) optimal matching rules via endowments (their non-manipulability by predonation corresponds to our transfer-proofness condition). Transfer-proofness is also related to the so-called "transfer paradox" (a trader can be hurt by accepting a predonation). Leontief (1936) is the first to demonstrate that the Walrasian rule is not immune to the transfer paradox for twoagent exchange economies. For two-agent economies, transfer-proofness is equivalent to being immune to the transfer-paradox. Thomson (1987) shows that transfer-proofness is compatible with efficiency and individual rationality in exchange economies.

We demonstrate that, similarly as in other models, efficient and individually rational rules are generally not immune to manipulations via endowments (Theorems 1, 2, and 3). However, we also identify some subclasses of exchange markets where these incompatibilities do not apply: for two agents with separable and responsive preferences, destructionproofness is compatible with efficiency and individual rationality (Proposition 1), and for two agents with unrestricted preferences, efficiency is stronger than transfer-proofness so that transfer-proofness is compatible with efficiency and individual rationality. ${ }^{3}$

\footnotetext{
${ }^{1}$ Alternatively, we consider myopic transfer-proofness by requiring that the recipient of the transfer experiences the transfer as weakly endowment improving.

${ }^{2}$ Thomson (1987) strengthens the former result by showing that the incompatibility persists on the restricted domain of homothetic preferences even if hiding-proofness is replaced by a weaker notion at which agents can consume only a positive percentage of what they hide no matter how small that percentage is.

${ }^{3}$ See also Proposition 2 in the Apendix for the compatibility of myopic transfer-proofness with efficiency and individual rationality.
} 


\section{The Model}

\subsection{Exchange Markets with Indivisible Objects}

Let $K$ be a set of heterogeneous objects containing at least two objects (we allow $|K|=\infty$ ). Let $2^{K}$ denote the set of all (possibly empty) subsets of $K$. To simplify notation, we omit the brackets when denoting subsets of $K$ and write, for instance, $x y z$ instead of $\{x, y, z\}$. Let $N \equiv\{1, \ldots, n\}$ be a finite set of agents containing at least two agents. Each agent $i \in N$ is endowed with a finite (possibly empty) set of objects $E_{i} \in 2^{K}$. No two agents own the same object(s). So, an endowment distribution $E \equiv\left(E_{1}, \ldots, E_{n}\right)$ is defined by (i) for all $i \in N,\left|E_{i}\right|<\infty$, (ii) $\bigcup_{i=1}^{n} E_{i} \in 2^{K}$, and (iii) for all $i, j \in N, E_{i} \cap E_{j}=\emptyset$ if $i \neq j$. Note that $\bigcup_{i=1}^{n} E_{i} \varsubsetneqq K$ is possible. We denote the set of all endowment distributions by $\mathcal{E}$.

Each agent $i \in N$ has complete and transitive preferences $R_{i}$ over $2^{K}$. The associated strict preference relation is denoted by $P_{i}$. Moreover, preferences are strict, that is, for all distinct subsets $S, S^{\prime} \in 2^{K}$, either $S P_{i} S^{\prime}$ or $S^{\prime} P_{i} S$. Thus, $S R_{i} S^{\prime}$ means that either $S P_{i} S^{\prime}$ or $S=S^{\prime}$.

An important preference restriction is separability: ${ }^{4}$ agent $i$ 's preferences are separable whenever she prefers $x$ to $\emptyset$ if and only if for any set $S$ not containing $x$ she prefers $S \cup x$ to $S$ : for all $S \subseteq K$ and all $x \in K \backslash S, x P_{i} \emptyset \Leftrightarrow(S \cup x) P_{i} S$. Together with strictness and completeness of preferences, this implies that for all $S \subseteq K$ and all $x \in K \backslash S$, $\emptyset P_{i} x \Leftrightarrow S P_{i}(S \cup x)$. Let $\mathcal{R}_{s}$ be the set of separable preference relations over $2^{K}$.

A preference restriction that is often combined with separability is responsiveness: agent $i$ 's preferences are responsive if, for any two sets that differ only in one object, agent $i$ prefers the set containing the more preferred object: for all $S \subseteq K$ and all $x, y \in K \backslash S$, $x P_{i} y \Rightarrow(S \cup x) P_{i}(S \cup y)$. Roth (1985) introduces this notion of responsiveness for college admission problems.

The last preference restriction we consider is additivity: agent $i$ 's preferences are $a d-$ ditive if there exists a function $u_{i}: K \rightarrow \mathbb{R}$ such that for all $S, S^{\prime} \in 2^{K}, S R_{i} S^{\prime} \Leftrightarrow$ $\sum_{k \in S} u_{i}(k) \geq \sum_{k \in S^{\prime}} u_{i}(k)$.

At various points, we consider the following four domains of preferences: the (otherwise) unrestricted domain of all strict preferences $\mathcal{R}_{u}$; the domain of separable preferences $\mathcal{R}_{s}$; the domain of separable and responsive preferences $\mathcal{R}_{s r}$; and the domain of additive preferences $\mathcal{R}_{a}$. Clearly, $\mathcal{R}_{a} \subsetneq \mathcal{R}_{s r} \subsetneq \mathcal{R}_{s} \subsetneq \mathcal{R}_{u}$. Whenever we introduce notation or concepts that apply to all preference domains, we use the generic preference domain $\mathcal{R}$. We denote a typical preference profile by $R=\left(R_{1}, R_{2}, \ldots, R_{n}\right)$ and the set of preference profiles by $\mathcal{R}^{N}$.

Thus, given a preference profile $R \in \mathcal{R}^{N}$ and an endowment distribution $E \in \mathcal{E}$, we denote an exchange market (with indivisible objects) by $(R, E)$. Since in the remainder of the article we assume that the preference profile remains fixed while endowment distributions may vary, we simply denote an exchange market by its endowment distribution $E \in \mathcal{E}$.

An allocation for an exchange market $E \in \mathcal{E}$ is a list $\left(S_{1}, \ldots, S_{n}\right)$ such that (i) each agent $i \in N$ receives some subset $S_{i} \subseteq \bigcup_{i=1}^{n} E_{i}$ and (ii) no two agents receive the same object: for all $i, j \in N, S_{i} \cap S_{j}=\emptyset$ if $i \neq j$. We allow for free disposal, that is, $\bigcup_{i=1}^{n} S_{i} \varsubsetneqq$ $\bigcup_{i=1}^{n} E_{i}$ is possible. Most of our results remain valid without free disposal (Lemma 1 is the exception).

\footnotetext{
${ }^{4}$ For the notion of separability we use here, we refer to Barberà, Sonnenschein and Zhou (1991).
} 


\subsection{Exchange Rules and their Properties}

An (exchange) rule is a function $\varphi$ that associates with each exchange market $E \in \mathcal{E}$ an allocation $\varphi(E)=\left(S_{i}\right)_{i \in N}$. Given $i \in N$, we call $\varphi_{i}(E)$ the allotment of agent $i$ at $\varphi(E)$.

Recall that in our model preferences are fixed. In addition, we will not consider any properties that link exchange markets on the basis of preference profiles. Therefore rules are only defined with respect to the given and fixed preference profile. In particular, when introducing dictatorial rules (see Examples 2-6 and 8-11) this means that the corresponding "dictatorial structure" may change across preference profiles.

Two standard requirements for rules are efficiency and individual rationality (agents find their allotments at least as good as their endowments):

Efficiency: For all $E \in \mathcal{E}$ there is no allocation $\left(S_{i}\right)_{i \in N}$ such that for all $i \in N, S_{i} R_{i} \varphi_{i}(E)$, with strict preference holding for some $j \in N$.

Individual Rationality: For all $E \in \mathcal{E}$ and all $i \in N, \varphi_{i}(E) R_{i} E_{i}$.

For all $E \in \mathcal{E}$, we denote the set of efficient allocations by $\mathcal{P}(E)$, the set of individually rational allocations by $\mathcal{I}(E)$, and the set of efficient and individually rational allocations by $\mathcal{P} \mathcal{I}(E)$.

Given that individual endowments are private information, an agent may manipulate the outcome to her advantage by hiding, destroying, or transferring part of her endowment.

Given an endowment distribution $E \in \mathcal{E}$, an agent $i \in N$, and a subset $E_{i}^{\prime} \subsetneq E_{i}$, we obtain the new endowment distribution $\left(E_{i}^{\prime}, E_{-i}\right)$ where agent $i$ hides part of her endowment by replacing agent $i$ 's endowment $E_{i}$ with $E_{i}^{\prime}$.

First, we consider hiding-proofness: if agent $i$ hides part of her endowment $E_{i}$ and pretends to only own $E_{i}^{\prime} \subsetneq E_{i}$, then she finds her original allotment $\varphi_{i}(E)$ at least as good as the set of objects $\varphi_{i}\left(E_{i}^{\prime}, E_{-i}\right) \cup\left(E_{i} \backslash E_{i}^{\prime}\right)$ she finally can consume.

Hiding-Proofness: For all $E \in \mathcal{E}$, all $i \in N$, and all $E_{i}^{\prime} \subsetneq E_{i}, \varphi_{i}(E) R_{i}\left[\varphi_{i}\left(E_{i}^{\prime}, E_{-i}\right) \cup\right.$ $\left.\left(E_{i} \backslash E_{i}^{\prime}\right)\right]$.

Since an agent could hide all of her endowment $\left(E_{i}^{\prime}=\emptyset\right)$, we deduce the following:

Lemma 1. For any profile of separable preferences, efficiency and hiding-proofness together imply individual rationality. ${ }^{5}$

Proof: Let $\varphi$ be efficient and hiding-proof. Let $E \in \mathcal{E}$ and $i \in N$. If $\varphi_{i}\left(\emptyset, E_{-i}\right)=\emptyset$, then by hiding-proofness, $\varphi_{i}(E) R_{i}\left(\varphi_{i}\left(\emptyset, E_{-i}\right) \cup E_{i}\right)=E_{i}$. If $\varphi_{i}\left(\emptyset, E_{-i}\right)=\left\{x_{1}, \ldots, x_{l-1}, x_{l}\right\} \neq \emptyset$, then by efficiency, separability, strictness, and free disposal, for all $k \in\{1, \ldots, l\}, x_{k} P_{i} \emptyset$. By separability, $\left(\varphi_{i}\left(\emptyset, E_{-i}\right) \cup E_{i}\right) P_{i}\left(\left\{x_{1}, \ldots, x_{l-1}\right\} \cup E_{i}\right) P_{i} \ldots P_{i}\left(x_{1} \cup E_{i}\right) P_{i} E_{i}$. Hence, by transitivity, $\left(\varphi_{i}\left(\emptyset, E_{-i}\right) \cup E_{i}\right) P_{i} E_{i}$. By hiding-proofness, $\varphi_{i}(E) R_{i}\left(\varphi_{i}\left(\emptyset, E_{-i}\right) \cup E_{i}\right)$. Thus, by transitivity, $\varphi_{i}(E) P_{i} E_{i}$. To summarize, for all $E \in \mathcal{E}$ and $i \in N, \varphi_{i}(E) R_{i} E_{i}$, i.e., $\varphi$ is individually rational.

If each object is desirable to each agent, that is, for all $i \in N$, and all $x \in K, x P_{i} \emptyset$, then Lemma 1 holds without efficiency (that is, hiding-proofness implies individual rationality). If each object is desirable to each agent, separability is equivalent to monotonicity, that is, for all $i \in N$, and all $S, S^{\prime} \in 2^{K}$, if $S \nsupseteq S^{\prime}$, then $S P_{i} S^{\prime}$. In fact, "hiding-proofness implies individual rationality" is a model-free observation if preferences are monotonic.

\footnotetext{
${ }^{5}$ Special thanks to Somdeb Lahiri for pointing out with an example that Lemma 1 is not true on $\mathcal{R}_{u}$.
} 
Lemma 1 may not be valid without free disposal (e.g., any efficient and hiding-proof rule for the free disposal setting can be easily extended to the "no-disposal" setting by assigning undesirable objects to a predetermined agent).

Second, we consider destruction-proofness: if an agent $i$ destroys part of her endowment $E_{i}$, thereby reducing it to $E_{i}^{\prime} \subsetneq E_{i}$, then she finds her original allotment $\varphi_{i}(E)$ at least as good as her new allotment $\varphi_{i}\left(E_{i}^{\prime}, E_{-i}\right)$.

Destruction-Proofness: For all $E \in \mathcal{E}$, all $i \in N$, and all $E_{i}^{\prime} \subsetneq E_{i}, \varphi_{i}(E) R_{i} \varphi_{i}\left(E_{i}^{\prime}, E_{-i}\right)$.

Given an endowment distribution $E \in \mathcal{E}$, agents $i, j \in N$, and a subset $E_{i}^{\prime} \subsetneq E_{i}$, we obtain the new endowment distribution $\left(E_{i}^{\prime}, E_{j}^{\prime}, E_{-i j}\right)$ where agent $i$ transfers part of her endowment, namely $E_{i} \backslash E_{i}^{\prime}$, to agent $j$ by replacing agent $i$ 's endowment $E_{i}$ with $E_{i}^{\prime}$ and agent $j$ 's endowment $E_{j}$ with $E_{j}^{\prime} \equiv E_{j} \cup E_{i} \backslash E_{i}^{\prime}$. We denote the exchange market that is obtained after agent $i$ transfers $E_{i} \backslash E_{i}^{\prime}$ to agent $j$ by $\left(E_{i}^{\prime}, E_{j}^{\prime}, E_{-i j}\right)$.

Third, we consider (farsighted) transfer-proofness: if agent $i$ transfers part of her endowment $E_{i}$ to another agent, say agent $j$, who is not worse off because of the transfer, i.e., $\varphi_{j}\left(E_{i}^{\prime}, E_{j}^{\prime}, E_{-i j}\right) R_{j} \varphi_{j}(E)$, then agent $i$ finds her original allotment $\varphi_{i}(E)$ at least as good as her new allotment $\varphi_{i}\left(E_{i}^{\prime}, E_{j}^{\prime}, E_{-i j}\right)$.

Transfer-Proofness: For all $E \in \mathcal{E}$, all $i, j \in N$, all $E_{i}^{\prime} \subsetneq E_{i}$, and $E_{j}^{\prime} \equiv E_{j} \cup E_{i} \backslash E_{i}^{\prime}$, if $\varphi_{j}\left(E_{i}^{\prime}, E_{j}^{\prime}, E_{-i j}\right) R_{j} \varphi_{j}(E)$, then $\varphi_{i}(E) R_{i} \varphi_{i}\left(E_{i}^{\prime}, E_{j}^{\prime}, E_{-i j}\right)$.

Obviously, efficiency implies transfer-proofness if $n=2$. Note that we only require that the recipient of the transfer experiences it as weakly allotment improving. We do not require that the recipient (weakly) prefers her endowment after the transfer to her endowment before the transfer. Hence, in the definition of transfer-proofness, we assume transfer recipients to be farsighted. By imposing the extra condition that transfer recipients experience the transfer as weakly endowment improving, we obtain the following weaker transfer-proofness property.

Weak Transfer-Proofness: For all $E \in \mathcal{E}$, all $i, j \in N$, all $E_{i}^{\prime} \subsetneq E_{i}$, and $E_{j}^{\prime} \equiv E_{j} \cup E_{i} \backslash E_{i}^{\prime}$, if $E_{j}^{\prime} R_{j} E_{j}$ and $\varphi_{j}\left(E_{i}^{\prime}, E_{j}^{\prime}, E_{-i j}\right) R_{j} \varphi_{j}(E)$, then $\varphi_{i}(E) R_{i} \varphi_{i}\left(E_{i}^{\prime}, E_{j}^{\prime}, E_{-i j}\right)$.

Obviously, transfer-proofness implies weak transfer-proofness. Note that if we only impose that transfer recipients experience the transfer as weakly endowment improving, we obtain a "myopic transfer-proofness" condition that is logically independent from our farsighted transfer-proofness conditions. All our results remain valid if we use myopic transfer-proofness instead of (farsighted) transfer-proofness. In the Appendix, we formally define myopic transfer-proofness and show how examples and proofs adapt if transfer proofness is replaced by myopic transfer-proofness.

As the following examples demonstrate, no direct relationship exists between hidingproofness, destruction-proofness, and (weak) transfer-proofness.

\section{Example 1. No-Trade Rule}

For any preference profile, the no-trade rule, a rule that assigns to each agent her endowment, is hiding-proof and individually rational. However, even for profiles with additive preferences, the no-trade rule may be neither destruction-proof, nor weakly transfer-proof, nor efficient. If $n=2$, then for any preference profile, the no-trade rule is transfer-proof as well.

Since later we show that no efficient and hiding-proof rule exists, it is not possible to find a rule that is efficient, hiding-proof, but not destruction-proof or not weakly transferproof. 


\section{Example 2. Serial Dictatorship Rule}

For any profile of separable preferences, a serial dictatorship rule, a rule that assigns to each agent in a serial way her most preferred set of objects (among the remaining objects), is destruction-proof, transfer-proof, and efficient. However, even for profiles with additive preferences, a serial dictatorship rule may be neither hiding-proof, nor individually rational.

We refer to Klaus and Miyagawa (2002) for a precise definition of serial dictatorship rules. For unrestricted preference profiles, a serial dictatorship rule may not be destructionproof (e.g., destroying an object may cause a predecessor to abstain from consuming other objects that she considers complementary to the destroyed one).

\section{Example 3. Conditional Serial Dictatorship Rule $\varphi^{c s d(x, E)}$}

A conditional serial dictatorship rule $\varphi^{\operatorname{csd}(x, E)}$ is defined as follows: Let $x \in K$ and $\varphi^{d}$, $\varphi^{d^{\prime}}$ be serial dictatorship rules such that for $\varphi^{d}$, lower-indexed agents come first and for $\varphi^{d^{\prime}}$, higher-indexed agents come first. For all $E \in \mathcal{E}$ such that $x \in \bigcup_{i \in N} E_{i}$, let $\varphi^{\operatorname{csd}(x, E)}(E) \equiv \varphi^{d}(E)$. For all $E \in \mathcal{E}$ such that $x \notin \bigcup_{i \in N} E_{i}$, let $\varphi^{\operatorname{csd}(x, E)}(E) \equiv \varphi^{d^{\prime}}(E)$.

For any profile of separable preferences, $\varphi^{c s d(x, E)}$ is efficient and transfer-proof. However, even for profiles with additive preferences, $\varphi^{\operatorname{csd}(x, E)}$ may be neither hiding-proof, nor individually rational, nor destruction-proof.

Example 4. Conditional Serial Dictatorship Rule $\varphi^{\operatorname{csd}\left(x, E_{1}\right)}$

A conditional serial dictatorship rule $\varphi^{\operatorname{csd}\left(x, E_{1}\right)}$ is defined as follows: Let $x \in K$ and $\varphi^{d}$, $\varphi^{d^{\prime}}$ be serial dictatorship rules such that for $\varphi^{d}$, lower-indexed agents come first and for $\varphi^{d^{\prime}}$, higher-indexed agents come first. For all $E \in \mathcal{E}$ such that $x \in E_{1}$, let $\varphi^{\operatorname{csd}\left(x, E_{1}\right)}(E) \equiv$ $\varphi^{d}(E)$. For all $E \in \mathcal{E}$ such that $x \notin E_{1}$, let $\varphi^{c s d\left(x, E_{1}\right)}(E) \equiv \varphi^{d^{\prime}}(E)$.

Let $n \geq 3$. Then, for any profile of separable preferences, $\varphi^{\operatorname{csd}\left(x, E_{1}\right)}$ is efficient and destruction-proof. However, even for profiles with additive preferences, $\varphi^{\operatorname{csd}\left(x, E_{1}\right)}$ may be neither hiding-proof, nor individually rational, nor weakly transfer-proof. If $n=2$, then for any profile of separable preferences, $\varphi^{c s d\left(x, E_{1}\right)}$ is transfer-proof as well.

\section{Results}

\subsection{Hiding-Proofness}

Theorem 1. For some profiles of additive preferences, no rule is efficient and hidingproof.

Proof: Let $\varphi$ be an efficient and hiding-proof rule. Let $N=\{1,2\}, E=\left(E_{1}, E_{2}\right)$ be such that $E_{1}=a b, E_{2}=c d$, and $\left(R_{1}, R_{2}\right) \in \mathcal{R}_{a}^{N}$ have the following utility representation

$$
\begin{array}{|l|l|}
\hline u_{1}(a)=5, & u_{2}(a)=6 \\
u_{1}(b)=2.1, & u_{2}(b)=3 \\
u_{1}(c)=3, & u_{2}(c)=1.1 \\
u_{1}(d)=4, & u_{2}(d)=4 \\
\hline
\end{array}
$$

Hence, by Lemma $1, \varphi$ is individually rational. The only efficient and individually rational allocations are $A=(a c, b d)$ and $B=(b c d, a)$. Hence, $\varphi(E) \in\{A, B\}$. 
Case 1: $\varphi(E)=A$. If agent 1 hides object $b$, the endowment distribution becomes $E^{1}=(a, c d)$ and the only efficient and individually rational allocation for the resulting exchange market is $A^{1}=(c d, a)$. So, $\varphi\left(E^{1}\right)=A^{1}$. Hence, agent 1 consumes $b c d$, which she prefers to $a c$, her allotment at $A$, in violation of hiding-proofness. Thus, $\varphi(E) \neq A$.

Case 2: $\varphi(E)=B$. If agent 2 hides object $d$, the endowment distribution becomes $E^{2}=(a b, c)$ and the only efficient and individually rational allocation for the resulting exchange market is $B^{1}=(a d, b)$. So, $\varphi\left(E^{2}\right)=B^{1}$. Hence, agent 2 consumes $b d$, which she prefers to $b c$, her allotment at $B$, in violation of hiding-proofness. Thus, $\varphi(E) \neq B$.

Cases 1 and 2 together show that for $n=2$, efficiency and hiding-proofness are incompatible. For $n>2$, we simply add agents who prefer their endowments to any other set of objects (including $\emptyset$ ). Since then only agents 1 and 2 trade with each other as specified above, the incompatibility of efficiency and hiding-proofness persists for $n>2$.

\subsection{Destruction-Proofness}

If we replace hiding-proofness by destruction-proofness, compatibility with efficiency and individual rationality is possible for two agents with separable and responsive preferences.

Let $N=\{1,2\}, R \in \mathcal{R}_{s r}^{N}$, and $E \in \mathcal{E}$. In order to present a rule satisfying the properties listed above, we introduce some notation. First, for $i \in N$, we obtain $\bar{E}_{i}$ by discarding each undesirable object $x$, that is, an object $x \in E_{i}$ such that $\emptyset P_{i} x$. Second, in order to preserve efficiency, we define the set $\tilde{E}_{i}$ by adding to $\bar{E}_{i}$ all objects that agent $j \neq i$ discarded, and that agent $i$ likes, that is, $\tilde{E}_{i} \equiv \bar{E}_{i} \cup\left\{x \in E_{j} \backslash \bar{E}_{j}: x P_{i} \emptyset\right\}$. Note that $\mathcal{P} \mathcal{I}(\tilde{E}) \subseteq \mathcal{P} \mathcal{I}(E)$.

\section{Example 5. Restricted (Serial) Dictatorship Rule $\varphi^{r d(i)}$}

Let $N=\{1,2\}$ and $i \in N$. For all $R \in \mathcal{R}_{s}^{N}$ and all $E \in \mathcal{E}, \varphi^{r d(i)}$ picks the unique best allocation for agent $i$ in $\mathcal{P} \mathcal{I}(\tilde{E})$. We call agent $i$ the restricted dictator. By construction, $\varphi^{r d(i)}$ is efficient and individually rational.

Next, we show that when preferences are separable and responsive, $\varphi^{r d(i)}$ is destructionproof. One can easily show that $\varphi^{r d(i)}$ is not hiding-proof.

Proposition 1. For two agents with separable and responsive preferences, restricted dictatorship rules are destruction-proof.

Proposition 1 only remains valid on $\mathcal{R}_{a}$ and $\mathcal{R}_{s r}$, but not on $\mathcal{R}_{s}$ and $\mathcal{R}_{u}$ (see Theorem 2). For $\mathcal{R}_{u}$, it is easy to see that destroying an object which is considered complementary by a previous restricted dictator, may induce this restricted dictator to choose a trade that is more advantageously for the agent who destroyed the object.

Proof: Let $N=\{1,2\}, \varphi=\varphi^{r d(1)}, R \in \mathcal{R}_{s r}^{N}$, and $E \in \mathcal{E}$. Note that by definition, no agent $i$ can benefit by destroying an undesirable object $x \in E_{i}$. Hence, it is without loss of

\footnotetext{
${ }^{6}$ For $n>2$ we can define restricted serial dictatorship rules $\tilde{\varphi}^{\text {rd( } \pi)}$, where $\pi$ denotes the ordering of "dictators." Similarly as before, we can derive an exchange market $\tilde{E}$ by first letting all agents discard of undesirable objects and then distributing them among the agents who would like to consume them (this distribution can, for instance, be done sequentially using $\pi$ ). Then, for all $R \in \mathcal{R}_{s}^{N}$ and $E \in \mathcal{E}$, the first dictator restricts the set $\mathcal{P} \mathcal{I}(\tilde{E})$ to all allocations where she receives her best allotment. Next, if several allocations are left over, the second dictator restricts the remaining set to all allocations where she receives her best allotment, etc.. In order to adjust restricted serial dictatorship rules if free disposal is not allowed, we simply assume that one of the agents has to keep any object that is undesirable for all agents.
} 
generality to assume that $E=\tilde{E}$. We prove that neither agent can benefit from destroying one of her objects. The proof that neither agent can benefit from destroying several objects follows by applying the "one-object-argument" for each object and invoking transitivity of preferences.

Case 1: Agent 1 destroys $x \in E_{1}$. Let $A \equiv \varphi(E)$ and $B \equiv \varphi\left(E_{1} \backslash x, E_{2}\right)$. Suppose $B_{1} P_{1} A_{1}$. By separability, $\left(B_{1} \cup x\right) P_{1} B_{1}$ and $\left(B_{1} \cup x, B_{2}\right) \in \mathcal{I}(E)$. Hence, there exists $C \in \mathcal{P} \mathcal{I}(E)$ such that $C_{1} R_{1}\left(B_{1} \cup x\right)$. Thus, $C_{1} P_{1} A_{1}$, which contradicts the assumption that $A$ is the best allocation for agent 1 in $\mathcal{P} \mathcal{I}(E)$.

Case 2: Agent 2 destroys $x \in E_{2}$. Let $A \equiv \varphi(E)$ and $B \equiv \varphi\left(E_{1}, E_{2} \backslash x\right)$. Suppose $B_{2} P_{2} A_{2}$. If $x \in A_{2}$, then $A_{2} R_{2} E_{2}$, which together with responsiveness implies that $A_{2} \backslash x R_{2} E_{2} \backslash x$. Then, $A \in \mathcal{P} \mathcal{I}(E)$ implies $\left(A_{1}, A_{2} \backslash x\right) \in \mathcal{P} \mathcal{I}\left(E_{1}, E_{2} \backslash x\right)$. Thus, by the definition of $\varphi, B_{1} R_{1} A_{1}$. This and $B_{2} P_{2} A_{2}$ contradict that $A \in \mathcal{P}(E)$. Hence, $x \in A_{1}$. Since $A \in \mathcal{P}(E), A_{1} P_{1}\left(B_{1} \cup x\right)$. By responsiveness, $A_{1} \backslash x P_{1} B_{1}$. Note that $\left(A_{1} \backslash x, A_{2}\right) \in$ $\mathcal{I}\left(E_{1}, E_{2} \backslash x\right)$. Hence, there exists $C \in \mathcal{P} \mathcal{I}\left(E_{1}, E_{2} \backslash x\right)$ such that $C_{1} P_{1} B_{1}$, which contradicts the assumption that $B$ is the best allocation for agent 1 in $\mathcal{P} \mathcal{I}\left(E_{1}, E_{2} \backslash x\right)$.

The following example describes a class of rules that are all efficient, individually rational, and destruction-proof.

Example 6. Restricted Conditional Dictatorship Rule $\varphi^{r c d\left(K^{\prime}, \bar{E}_{1}\right)}$

Let $N=\{1,2\}$ and $K^{\prime} \subseteq K$. For all $R \in \mathcal{R}_{s r}^{N}$ and all $E \in \mathcal{E}$ such that $K^{\prime} \subseteq \bar{E}_{1}$, $\varphi^{r c d\left(K^{\prime}, \bar{E}_{1}\right)}(E) \equiv \varphi^{r d(1)}(E)$. For all $R \in \mathcal{R}_{s r}^{N}$ and all $E \in \mathcal{E}$ such that $K^{\prime} \nsubseteq \bar{E}_{1}$, $\varphi^{r c d\left(K^{\prime}, \bar{E}_{1}\right)}(E) \equiv \varphi^{r d(2)}(E)$. Then, $\varphi^{r c d\left(K^{\prime}, \bar{E}_{1}\right)}$ is efficient, individually rational, and destruction-proof.

Many other restricted conditional dictatorship rules that are destruction-proof and are similar to those in Example 6 can be constructed. For instance, one can condition the choice of the restricted dictator differently, e.g., by $K^{\prime} \cap \bar{E}_{1} \neq \emptyset$ instead of $K^{\prime} \subseteq \bar{E}_{1}$. Hence, the class of rules that are efficient, individually rational, and destruction-proof for two agents with separable and responsive preferences is very large.

The next example demonstrates that for more than two agents, a restricted serial dictatorship rule may be manipulable by destruction. This result holds for any subdomain of $\mathcal{R}_{s}$ that includes the domain of additive preferences $\mathcal{R}_{a}$, in particular, for $\mathcal{R}_{a}, \mathcal{R}_{s r}$, and $\mathcal{R}_{s}$ (recall that our definition of a restricted serial dictatorship rules only applies to separable preferences so that we cannot make any statements about $\mathcal{R}_{u}$ ).

Example 7. Let $N=\{1,2,3\}, E=\left(E_{1}, E_{2}, E_{3}\right)$ be such that $E_{1}=a, E_{2}=b c, E_{3}=d e$, and $\left(R_{1}, R_{2}, R_{3}\right) \in \mathcal{R}_{a}^{N}$ have the following utility representation

\begin{tabular}{|l|l|l|}
\hline$u_{1}(a)=1$, & $u_{2}(a)=5$, & $u_{3}(a)=7$, \\
$u_{1}(b)=8$, & $u_{2}(b)=4$, & $u_{3}(b)=6$, \\
$u_{1}(c)=5$, & $u_{2}(c)=2$, & $u_{3}(c)=1.1$ \\
$u_{1}(d)=10.5$, & $u_{2}(d)=8$, & $u_{3}(d)=3$ \\
$u_{1}(e)=0.1$, & $u_{2}(e)=1.5$, & $u_{3}(e)=2.3$. \\
\hline
\end{tabular}

If agent 1 is the restricted dictator, then the restricted serial dictatorship rule picks $(c d, a e, b)$. However, if agent 3 destroys object $e$, for the resulting exchange market, the restricted serial dictatorship rule picks $(b c, d, a)$. Hence, agent 3 consumes $a$, which she strictly prefers to $b$, in violation of destruction-proofness. 
It is an open question whether for more than two agents with additive, or separable and responsive preferences, efficient, individually rational, and destruction-proof rules exist. If preferences are "only" separable, then we can establish the incompatibility of efficiency, individual rationality, and destruction-proofness for any number of agents.

Theorem 2. For some profiles of separable preferences, no rule is efficient, individually rational, and destruction-proof.

Proof: Let $\varphi$ be an efficient, individually rational, and destruction-proof rule. Let $N=$ $\{1,2\}, E=\left(E_{1}, E_{2}\right)$ be such that $E_{1}=a b, E_{2}=c d e$, and $\left(R_{1}, R_{2}\right) \in \mathcal{R}_{s}^{N}$ be as in Table 1. The underline symbol '.' in each allotment indicates that the corresponding object is not in the allotment. This presentation makes it easier to verify that both preference relations are separable. In order to save space, each linear ordering is listed in two columns. Once the reader reaches the bottom of the first column, she should continue from the top of the second column. The important entries are marked in boldface and the endowments are underlined. ${ }^{7}$

\begin{tabular}{|c|c|c|c|c|c|c|c|c|c|c|c|c|c|c|c|c|c|c|c|}
\hline \multicolumn{10}{|c|}{$R_{1}$} & \multicolumn{10}{|c|}{$R_{2}$} \\
\hline$a$ & $\bar{~} \bar{b}$ & $c$ & & $e$ & - & $\bar{~} \bar{b}$ & $c$ & - & $\overline{\bar{e}}$ & & $\overline{\bar{b}}$ & 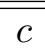 & $\overline{c d}$ & $\overline{\bar{e}}$ & $a$ & $\overline{\bar{b}}$ & - & - & \\
\hline$a$ & $b$ & $c$ & $d$ & - & - & $b$ & - & $d$ & $e$ & 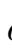 & $b$ & $c$ & $d$ & - & $a$ & - & $c$ & - & - \\
\hline$a$ & $b$ & $c$ & - & $e$ & $a$ & - & - & $d$ & - & 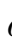 & $b$ & $c$ & - & $e$ & $a$ & - & _ & $d$ & - \\
\hline$a$ & $b$ & - & $d$ & $e$ & $a$ & - & - & - & $e$ & & $b$ & - & $d$ & $e$ & $a$ & - & - & - & $e$ \\
\hline$a$ & - & $c$ & $d$ & $e$ & $a$ & - & - & - & - & 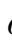 & _ & $c$ & $d$ & $e$ & $a$ & - & _ & - & - \\
\hline - & $b$ & $c$ & $d$ & $e$ & - & $b$ & $c$ & - & - & & $b$ & $c$ & $d$ & $e$ & - & - & $\underline{c}$ & $\underline{d}$ & $\underline{e}$ \\
\hline _ & - & $c$ & $d$ & $e$ & - & $b$ & - & $d$ & - & & $b$ & $c$ & - & - & - & $b$ & $c$ & - & _ \\
\hline$a$ & $b$ & $c$ & - & - & - & $b$ & - & - & $e$ & & $b$ & - & $d$ & - & - & $b$ & - & - & $e$ \\
\hline$a$ & $b$ & - & $d$ & - & - & - & $c$ & $d$ & - & & $b$ & - & - & $e$ & - & - & $c$ & $d$ & - \\
\hline$a$ & $b$ & - & - & $e$ & - & - & $c$ & - & $e$ & & - & $c$ & $d$ & - & - & - & $c$ & - & $e$ \\
\hline$a$ & - & $c$ & $d$ & - & - & - & - & $d$ & $e$ & & - & $c$ & - & $e$ & - & - & - & $d$ & $e$ \\
\hline$a$ & - & $c$ & - & $e$ & - & $b$ & - & - & - & & - & - & $d$ & $e$ & - & $b$ & - & - & - \\
\hline$a$ & - & $c$ & - & - & - & - & $c$ & - & - & & $b$ & $c$ & $d$ & - & - & - & $c$ & - & - \\
\hline$\underline{a}$ & $\underline{b}$ & - & - & - & - & - & - & $d$ & - & & $b$ & $c$ & - & $e$ & - & - & - & $d$ & - \\
\hline$a$ & - & - & $d$ & $e$ & - & - & - & - & $e$ & & $b$ & - & $d$ & $e$ & - & - & - & - & $e$ \\
\hline - & $b$ & $c$ & $d$ & - & - & - & - & - & - & & $b$ & - & $d$ & - & - & - & - & - & _ \\
\hline
\end{tabular}

Table 1: Complete separable preferences in the proof of Theorem 2.

We now explain how one can reduce the preference table to allotments that can occur at efficient and individually rational allocations. First, by individual rationality, for each agent, we can delete all allotments that are ranked below her endowment. Next, by individual rationality, agent 1 has to receive at least two objects and, agent 2 has to receive $a$ or at least two objects. Hence, we can delete all allotments containing more than three objects, except bcde for agent 1 . Finally, at any efficient allocation all objects must be assigned. Thus, we can delete allotments from an agent's preference relation if the remaining objects are not individually rational for the other agent. For example, since $b c$

\footnotetext{
${ }^{7}$ Note that the ranking of boldfaced entries in Table 1 contains the information on preferences we use in the proof. We then constructed Table 1 as a separable extension of these preferences.
} 
is not individually rational for agent 1 , agent 2 will never receive ade and we can delete the associated entry in Table 1. Hence, by efficiency and individual rationality, we can focus on the part of the preferences depicted in Table 2 .

\begin{tabular}{|ccccc||ccccc|}
\hline \multicolumn{5}{|c||}{$R_{1}$} & \multicolumn{5}{c|}{$R_{2}$} \\
\hline \hline- & $b$ & $c$ & $d$ & $e$ & - & $b$ & - & $d$ & $e$ \\
- & - & $c$ & $d$ & $e$ & - & $b$ & - & $d$ & - \\
$a$ & - & $c$ & - & $e$ & $a$ & $b$ & - & - & - \\
$a$ & - & $c$ & - & - & $a$ & - & - & - & - \\
$a$ & $b$ & - & - & - & - & - & $c$ & $d$ & $e$ \\
\hline
\end{tabular}

Table 2: Relevant entries in Table 1 after taking efficiency and individual rationality into account.

Note that $E$ is not efficient. Hence, according to Table 2, the only efficient and individually rational allocations are $A=(b c d e, a), B=(c d e, a b), C=(a c e, b d)$, and $D=(a c, b d e)$. Hence, $\varphi(E) \in\{A, B, C, D\}$.

Case 1: $\varphi(E) \in\{A, B\}$. If agent 2 destroys object $e$, the endowment distribution becomes $E^{1}=(a b, c d)$. It is easy to check that the only efficient and individually rational allocation for the resulting exchange market is $A^{1}=(a c, b d)$. So, $\varphi\left(E^{1}\right)=A^{1}$. Hence, agent 2 consumes $b d$, which she prefers to $a$, her allotment at $A$; and to $a b$, her allotment at $B$, in violation of destruction-proofness. Thus, $\varphi(E) \notin\{A, B\}$.

Case 2: $\varphi(E) \in\{C, D\}$. If agent 1 destroys object $b$, the endowment distribution becomes $E^{2}=(a, c d e)$. It is easy to check that the only efficient and individually rational allocation for the resulting exchange market is $C^{1}=(c d e, a)$. So, $\varphi\left(E^{2}\right)=C^{1}$. Hence, agent 1 consumes $c d e$, which she prefers to ace, her allotment at $C$; and to $a c$, her allotment at $D$, in violation of destruction-proofness. Thus, $\varphi(E) \notin\{C, D\}$.

Cases 1 and 2 together show that for $n=2$, efficiency, individual rationality, and destruction-proofness are incompatible. For $n>2$, we simply add agents who prefer their endowments to any other set of objects (including $\emptyset$ ). Since then only agents 1 and 2 trade with each other as specified above, the incompatibility of efficiency, individual rationality, and destruction-proofness persists for $n>2$.

\subsection{Transfer-Proofness}

For two agents, efficiency implies transfer-proofness. Hence, when $n=2$, for any preference profile, any efficient and individually rational rule is transfer-proofness.

It is an open question whether for more than two agents with either additive, or separable and responsive preferences, efficient, individually rational, and weakly transferproof rules exist. However, for more than two agents with separable preferences, these properties are not compatible.

Theorem 3. For some profiles of separable preferences and at least three agents, no rule is efficient, individually rational, and weakly transfer-proof. 
Proof: Let $\varphi$ be an efficient, individually rational, and transfer-proof rule. Let $N=$ $\{1,2,3\}, E=\left(E_{1}, E_{2}, E_{3}\right)$ be such that $E_{1}=a b, E_{2}=c d, E_{3}=e f$, and $\left(R_{1}, R_{2}, R_{3}\right) \in \mathcal{R}_{s}^{N}$ be as in Table 3 (the structure is as in Table 1). The important entries are marked in bold face and the endowments are underlined. ${ }^{8}$

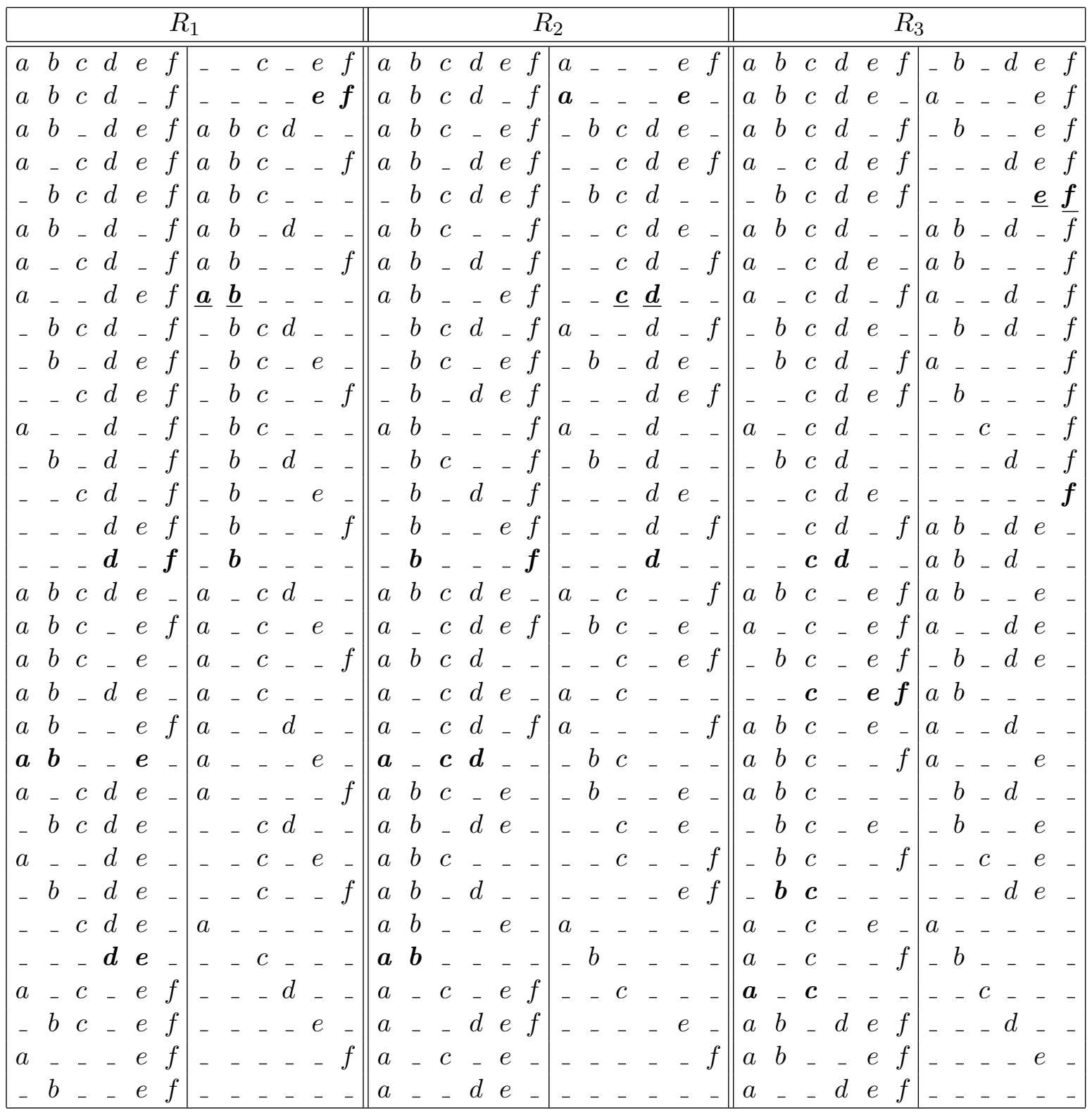

Table 3: Complete separable preferences in the proof of Theorem 3.

We now explain how one can reduce the preference table to allotments that can occur at efficient and individually rational allocations. First, by individual rationality, for each agent, we can delete all allotments that are ranked below her endowment. All individually

\footnotetext{
${ }^{8}$ Note that the ranking of boldfaced entries in Table 3 contains the information on preferences we use in the proof. We then constructed Table 3 as a separable extension of these preferences.
} 
rational allotments that are left contain at least two objects for each agent. Hence, we can delete all allotments containing more than two objects. Thus, by individual rationality, we can focus on the part of the preferences depicted in Table 4.

\begin{tabular}{|c|c|c|c|c|c|c|c|c|c|c|c|c|c|c|c|c|}
\hline & \multicolumn{3}{|c|}{$R_{1}$} & & \multicolumn{6}{|c|}{$R_{2}$} & \multicolumn{5}{|c|}{$R_{3}$} & \\
\hline- & & - & & & - & & - & & - & & - & - & & & & - \\
\hline - & - & - & & & $a$ & $b$ & - & - & - & - & - & $b$ & $c$ & & & - \\
\hline- & - & - & & & $a$ & - & - & - & $e$ & - & $a$ & - & $c$ & & - & - \\
\hline$a$ & $b$ & - & & & - & - & $c$ & $d$ & - & - & - & - & - & & $e$ & $f$ \\
\hline
\end{tabular}

Table 4: Relevant entries in Table 3 after taking individual rationality into account.

Note that $E$ is not efficient. Hence, according to Table 4 , the only efficient and individually rational allocations are $A=(d f, a e, b c), B=(d e, b f, a c)$, and $C=(e f, a b, c d)$. Hence, $\varphi(E) \in\{A, B, C\}$.

Case 1: $\varphi(E)=A$. If agent 2 transfers object $c$ to agent 3 , the endowment distribution becomes $E^{1}=(a b, d, c e f)$. We now explain how one can reduce the preference table to allotments that can occur at efficient and individually rational allocations for exchange market $E^{1}$. First, by individual rationality, for each agent, we can delete all allotments that are ranked below her endowment. Next, by individual rationality, agent 1 has to receive at least two objects; agent 2 has to keep her endowment or receive at least two objects, and agent 3 has to receive $c d$ or at least three objects. Thus, agent 1 can receive three objects only if none of them are $c$ or $d$, and agent 2 can receive two objects only if none of them are $c$ or $d$. Hence, by individual rationality, we can focus on the part of the preferences depicted in Table 5 .

\begin{tabular}{|c|c|c|c|c|c|c|c|c|c|c|c|c|c|c|c|}
\hline & \multicolumn{4}{|c|}{$R_{1}$} & \multicolumn{5}{|c|}{$R_{2}$} & \multicolumn{6}{|c|}{$R_{3}$} \\
\hline & - & $-\quad 0$ & - & & $-b$ & - & - & - & & & - & $c$ & & & \\
\hline$a$ & $b$ & - & $e$ & - & $\begin{array}{ll}a & b\end{array}$ & - & $\ldots$ & - & - & & $b$ & $c$ & $d$ & - & - \\
\hline - & - & -0 & $e$ & - & $a \quad-$ & - & _. & $e$ & - & - & - & $c$ & $d$ & $e$ & - \\
\hline$a$ & - & - & $e$ & $f$ & $-\quad-$ & & & - & - & - & - & $c$ & $d$ & - & $f$ \\
\hline - & $b$ & - & $e$ & $f$ & & & & & & - & - & $c$ & $d$ & - & - \\
\hline - & - & - & $e$ & $f$ & & & & & & - & & & & & $f$ \\
\hline$a$ & $b$ & - & - & $f$ & & & & & & & & & & & \\
\hline$a$ & $b$ & - & _ & - & & & & & & & & & & & \\
\hline
\end{tabular}

Table 5: Case 1, relevant entries in Table 3 after taking individual rationality into account.

Note that $E^{1}$ is not efficient. Hence, according to Table 5, the only efficient and individually rational allocation for the resulting exchange market is $C=(e f, a b, c d)$. So, $\varphi\left(E^{1}\right)=C$. Hence, agent 2 consumes $a b$, which she prefers to $a e$, her allotment at $A$, in violation of transfer-proofness. Since agent 3 receives $c d$, which she prefers to $b c$, we also have a contradiction to weak transfer-proofness. Thus, $\varphi(E) \neq A$.

Case 2: $\varphi(E)=B$. If agent 3 transfers object $e$ to agent 1 , the endowment distribution becomes $E^{2}=(a b e, c d, f)$. Using similar arguments as in Case 1, we can focus on the part of the preferences depicted in Table 6 . 


\begin{tabular}{|c|c|c|c|c|c|c|c|c|c|c|c|c|c|c|c|c|}
\hline \multicolumn{6}{|c|}{$R_{1}$} & \multicolumn{6}{|c|}{$R_{2}$} & \multicolumn{5}{|c|}{$R_{3}$} \\
\hline$a$ & - & & & - & & - & & - & - & - & $f$ & & $b$ & & & $-\quad-$ \\
\hline- & $b$ & - & $d$ & - & & $a$ & & $c$ & - & - & - & $a$ & - & $c$ & - & - $\quad-$ \\
\hline- & - & $c$ & & - & & $a$ & $b$ & - & - & $e$ & - & - & - & - & - & $-f$ \\
\hline- & - & - & $d$ & $e$ & $f$ & $a$ & $b$ & - & - & - & - & & & & & \\
\hline- & - & - & & - & & $a$ & - & $c$ & - & $e$ & - & & & & & \\
\hline$a$ & $b$ & - & - & $e$ & - & $a$ & - & - & - & $\epsilon$ & - & & & & & \\
\hline & & & & & & - & - & $c$ & $d$ & - & _ & & & & & \\
\hline
\end{tabular}

Table 6: Case 2, relevant entries in Table 3 after taking individual rationality into account.

Note that $E^{2}$ is not efficient. Hence, according to Table 6 , the only efficient and individually rational allocation for the resulting exchange market is $A=(d f, a e, b c)$. So, $\varphi\left(E^{2}\right)=A$. Hence, agent 3 consumes $b c$, which she prefers to $a c$, her allotment at $B$, in violation of transfer-proofness. Since agent 1 receives $d f$, which she prefers to $d e$, we also have a contradiction to weak transfer-proofness. Thus, $\varphi(E) \neq B$.

Case 3: $\varphi(E)=C$. If agent 1 transfers object $a$ to agent 2, the endowment distribution becomes $E^{3}=(b, a c d$, ef $)$. Using similar arguments as in Case 1, we can focus on the part of the preferences depicted in Table 7 .

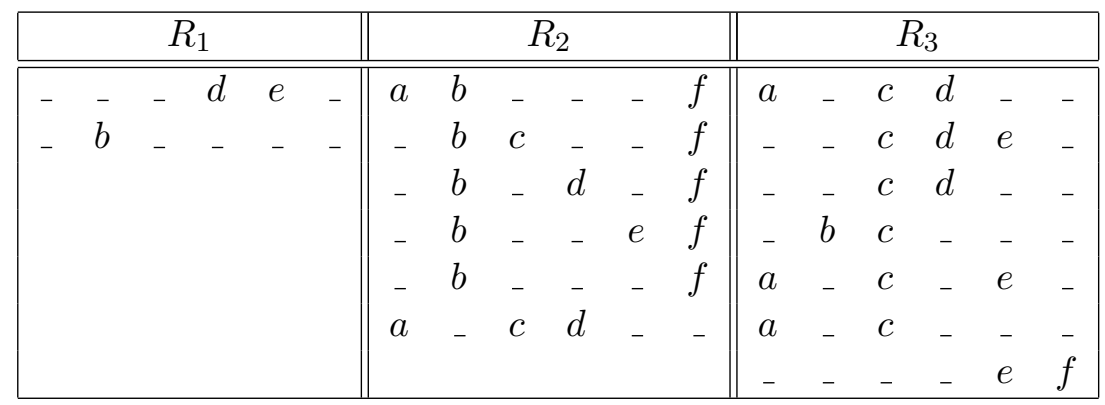

Table 7: Case 3, relevant entries in Table 3 after taking individual rationality into account.

Note that $E^{3}$ is not efficient. Hence, according to Table 7 , the only efficient and individually rational allocation for the resulting exchange market is $B=(d e, b f, a c)$. So, $\varphi\left(E^{3}\right)=B$. Hence, agent 1 consumes de, which she prefers to $e f$, her allotment at $C$, in violation of transfer-proofness. Since agent 2 receives $b f$, which she prefers to $a b$, we also have a contradiction to weak transfer-proofness. Thus, $\varphi(E) \neq C$.

Cases 1, 2, and 3 together show that efficiency, individual rationality, and weak transferproofness are incompatible for three agents. For $n>3$, we simply add agents who prefer their endowments to any other set of objects (including $\emptyset$ ). Since then, only agents 1,2 , and 3 trade with each other as specified above, the incompatibility of efficiency, individual rationality, and weak transfer-proofness persists for $n>3$. 


\section{Conclusion}

In this paper, we show that for separable preferences, efficient and individually rational rules are generally not immune to manipulations via endowments (Theorems 1, 2, and 3). An exception is the compatibility of efficiency, individual rationality and transferproofness in the two-agent case (efficiency then implies transfer-proofness). If in addition to separability we impose responsiveness, we obtain compatibility of efficiency, individual rationality, and destruction-proofness in the two-agent case (Proposition 1). We conjecture that the "dividing line" between compatibility and incompatibility lies between the preference domains $\mathcal{R}_{s r}$ and $\mathcal{R}_{s}$. However, two interesting questions we could not answer are:

Are efficiency, individual rationality, and destruction-proofness compatible for more than two agents with separable and responsive (additive) preferences?

Are efficiency, individual rationality, and (weak) transfer-proofness compatible for more than two agents with separable and responsive (additive) preferences?

A reason why it is not easy to answer these questions is that as the numbers of agents and objects become larger, it gets more difficult to determine the set of efficient and individually rational allocations.

\section{Appendix: Myopic Transfer-Proofness}

As an alternative transfer-proofness condition we now consider myopic transfer-proofness: if agent $i$ transfers part of her endowment $E_{i}$ to another agent, say agent $j$, who experiences the transfer as weakly endowment improving, thereby reducing her endowment to $E_{i}^{\prime} \subsetneq E_{i}$, and expanding agent $j$ 's endowment to $E_{j}^{\prime} \supsetneq E_{j}$ such that $E_{j}^{\prime} R_{j} E_{j}$, then agent $i$ finds her original allotment $\varphi_{i}(E)$ at least as good as her new allotment $\varphi_{i}\left(E_{i}^{\prime}, E_{j}^{\prime}, E_{-i j}\right)$.

Myopic Transfer-Proofness: For all $E \in \mathcal{E}$, all $i, j \in N$, all $E_{i}^{\prime} \subsetneq E_{i}$, and $E_{j}^{\prime} \equiv$ $E_{j} \cup E_{i} \backslash E_{i}^{\prime}$, if $E_{j}^{\prime} R_{j} E_{j}$, then $\varphi_{i}(E) R_{i} \varphi_{i}\left(E_{i}^{\prime}, E_{j}^{\prime}, E_{-i j}\right)$.

Obviously, myopic transfer-proofness implies weak transfer-proofness. Furthermore, the serial dictatorship rules and conditional serial dictatorship rules $\varphi^{c s d(x, E)}$ are myopic transfer-proof (see Examples 2 and 3). Clearly, the no-trade rule and the conditional serial dictatorship rules $\varphi^{c s d\left(x, E_{1}\right)}$ are not myopic transfer-proof (see Examples 1 and 4). Hence, myopic transfer-proofness is logically independent of hiding-proofness and destructionproofness.

Next, we consider the independence of myopic transfer-proofness and transferproofness. There are two cases of underlying separable preference profiles where myopic transfer-proofness and transfer-proofness are not logically independent.

The first case is when no object is desirable, i.e., for all $x \in K$ and all $i \in N, \emptyset P_{i} x$. In this case, any rule is myopic transfer-proof by definition and therefore transfer-proofness trivially implies myopic transfer-proofness.

The following rule shows that if a desirable object exists, then transfer-proofness does not necessarily imply myopic transfer-proofness. Without loss of generality, let $x^{+}$be a "desirable object" for agent 1 , i.e., $x^{+} P_{1} \emptyset$. 
Example 8. Conditional Serial Dictatorship Rule $\varphi^{\operatorname{csd}\left(x^{+}, E_{1},\{1,2\}\right)}$

A conditional serial dictatorship rule $\varphi^{c s d\left(x^{+}, E_{1},\{1,2\}\right)}$ is defined as follows: Let $x^{+} \in K$ be such that $x^{+} P_{1} \emptyset$, and $\varphi^{d}, \varphi^{d^{\prime}}$ be serial dictatorship rules such that for $\varphi^{d}$, lower-indexed agents come first and for $\varphi^{d^{\prime}}$, the order of agents 1 and 2 is switched and for the rest, lowerindexed agents come first. For all $E \in \mathcal{E}$ such that $x^{+} \in E_{1}$, let $\varphi^{\operatorname{csd}\left(x^{+}, E_{1},\{1,2\}\right)}(E) \equiv$ $\varphi^{d^{\prime}}(E)$. For all $E \in \mathcal{E}$ such that $x^{+} \notin E_{1}$, let $\varphi^{\operatorname{csd}\left(x^{+}, E_{1},\{1,2\}\right)}(E) \equiv \varphi^{d}(E)$. On $\mathcal{R}_{a}$, $\mathcal{R}_{s r}$, and $\mathcal{R}_{s}, \varphi^{c s d\left(x^{+}, E_{1},\{1,2\}\right)}$ is efficient and transfer-proof, but not myopic transfer-proof (agent 2 may benefit from transferring object $x^{+}$to agent 1 ).

The second case when transfer-proofness and myopic transfer-proofness are not logically independent occurs when all objects are desirable, i.e., for all $x \in K$ and all $i \in N$, $x P_{i} \emptyset$. In this case, all transfers are weakly endowment improving for the recipient and therefore myopic transfer-proofness implies transfer-proofness.

The following rule shows that if an undesirable object exists, then myopic transferproofness does not necessarily imply transfer-proofness. Without loss of generality, let $x^{-}$ be an "undesirable object" for agent 1 , i.e., $\emptyset P_{1} x^{-}$.

Example 9. Conditional Serial Dictatorship Rule $\varphi^{\operatorname{csd}\left(x^{-}, E_{1},\{1,2\}\right)}$

A conditional serial dictatorship rule $\varphi^{c s d\left(x^{-}, E_{1},\{1,2\}\right)}$ is defined as follows: Let $x^{-} \in K$ be such that $\emptyset P_{1} x^{-}$, and $\varphi^{d}, \varphi^{d^{\prime}}$ be serial dictatorship rules such that for $\varphi^{d}$, lowerindexed agents come first, and for $\varphi^{d^{\prime}}$, starting with agent 3 lower-indexed agents come first and then agents 1 and 2 come at the end of this order. For all $E \in \mathcal{E}$ such that $x^{-} \in E_{1}$, let $\varphi^{\operatorname{csd}\left(x^{-}, E_{1},\{1,2\}\right)}(E) \equiv \varphi^{d}(E)$. For all $E \in \mathcal{E}$ such that $x^{-} \notin E_{1}$, let $\varphi^{\operatorname{csd}\left(x^{-}, E_{1},\{1,2\}\right)}(E) \equiv \varphi^{d^{\prime}}(E)$. Let $n \geq 3$. Then, for any separable preference profile, $\varphi^{c s d\left(x^{-}, E_{1},\{1,2\}\right)}$ is efficient and myopic transfer-proof. However, even for profiles with additive preferences, $\varphi^{c s d\left(x^{-}, E_{1},\{1,2\}\right)}$ may not be transfer-proof (agents 1 and 2 both may benefit if agent 2 transfers object $x^{-}$to agent 1 ). If $n=2$, then for any separable preference profile, $\varphi^{c s d\left(x^{-}, E_{1},\{1,2\}\right)}$ is transfer-proof as well.

All our results remain valid if we use myopic transfer-proofness instead of (farsighted) transfer-proofness. Even though efficiency does not imply myopic transfer-proofness when $n=2$, we can still establish the compatibility of myopic transfer-proofness with efficiency and individual rationality for two agents. In fact, restricted serial dictatorship rules (defined in Section 3.2 on the domain of separable preferences in Example 5 and Footnote 5) are myopic transfer-proof.

We extend the definition of restricted (serial) dictatorship rules to the domain of unrestricted preferences $\mathcal{R}_{u}$. Let $N=\{1,2\}, R \in \mathcal{R}_{u}^{N}$, and $E \in \mathcal{E}$. For all $j \in N$, let $\bar{E}_{j}$ be the most preferred subset of $E_{j}$ for agent $j$, that is, for all $S \subseteq E_{j}, \bar{E}_{j} R_{j} S$.

\section{Example 10. Restricted (Serial) Dictatorship Rule $\varphi^{r d(i)}$}

Let $N=\{1,2\}$ and $i \in N$. For all $R \in \mathcal{R}_{u}^{N}$ and all $E \in \mathcal{E}, \varphi^{r d(i)}$ picks the unique best allocation for agent $i$ in $\mathcal{P} \mathcal{I}(E)$ that is individually rational for agent $j \neq i$ with respect to $\bar{E}_{j}$, that is, $\varphi_{j}^{r d(i)}(E) R_{j} \bar{E}_{j}$. By construction, $\varphi^{r d(i)}$ is efficient and individually rational. $\diamond$

Next, we show that $\varphi^{r d(i)}$ is myopic transfer-proof.

Proposition 2. For two agents with unrestricted preferences, restricted dictatorship rules are myopic transfer-proof. 
Proposition 2 remains valid on $\mathcal{R}_{a}, \mathcal{R}_{s r}, \mathcal{R}_{s}$, and $\mathcal{R}_{u}$.

Proof: Let $N=\{1,2\}, \varphi=\varphi^{r d(1)}, R \in \mathcal{R}_{u}^{N}$, and $E \in \mathcal{E}$. We prove that neither agent can benefit from transferring one of her objects to the other agent. The proof that neither agent can benefit from transferring several objects follows by applying the "one-objectargument" for each object and invoking transitivity of preferences.

Case 1: Agent 1 transfers $x \in E_{1}$ to agent 2. Let $E_{2}^{\prime} \equiv\left(E_{2} \cup x\right) R_{2} E_{2}$. Let $A \equiv \varphi(E)$ and $B \equiv \varphi\left(E_{1} \backslash x, E_{2}^{\prime}\right)$. Suppose $B_{1} P_{1} A_{1}$. Since $\bar{E} \in \mathcal{I}(E)$, by the definition of $\varphi, A_{1} R_{1} \bar{E}_{1}$. By individual rationality, $B_{2} R_{2} \bar{E}_{2}^{\prime} R_{2} E_{2}^{\prime}$. Note also that $\bar{E}_{2}^{\prime} R_{2} \bar{E}_{2}$. Then, by transitivity, $B_{1} P_{1} \bar{E}_{1}$ and $B_{2} R_{2} \bar{E}_{2}$. Hence, there exists $C \in \mathcal{P} \mathcal{I}(E)$ such that $C_{1} R_{1} B_{1}$ and $C_{2} R_{2} B_{2}$. Thus, $C_{1} P_{1} A_{1}$ and $C_{2} R_{2} \bar{E}_{2}$, which contradicts the assumption that $A$ is the best allocation for agent 1 in $\mathcal{P} \mathcal{I}(E)$.

Case 2: Agent 2 transfers $x \in E_{2}$ to agent 1 . Let $\left(E_{1} \cup x\right) R_{1} E_{1}$ and $E_{2}^{\prime} \equiv E_{2} \backslash x$. Let $A \equiv \varphi(E)$ and $B \equiv \varphi\left(E_{1} \cup x, E_{2}^{\prime}\right)$. Suppose $B_{2} P_{2} A_{2}$. Then, by efficiency, $A_{1} P_{1} B_{1}$. By individual rationality, $B_{1} R_{1}\left(E_{1} \cup x\right)$. By the definition of $\varphi^{r}, A_{2} R_{2} \bar{E}_{2}$. Note that $\bar{E}_{2} R_{2} \bar{E}_{2}^{\prime}$. Then, by transitivity, $A_{1} P_{1}\left(E_{1} \cup x\right)$ and $A_{2} R_{2} \bar{E}_{2}^{\prime}$. Hence, there exists $C \in \mathcal{P} \mathcal{I}\left(E_{1} \cup x, E_{2}^{\prime}\right)$ such that $C_{1} R_{1} A_{1}$ and $C_{2} R_{2} A_{2}$. Thus, $C_{1} P_{1} B_{1}$ and $C_{2} R_{2} \bar{E}_{2}^{\prime}$, which contradicts the assumption that $B$ is the best allocation for agent 1 in $\mathcal{P} \mathcal{I}\left(E_{1} \cup x, E_{2}^{\prime}\right)$.

The following example describes a class of rules that are all efficient, individually rational, and myopic transfer-proof.

Example 11. Restricted Conditional Dictatorship Rule $\varphi^{r c d(x, \tilde{E})}$

Let $N=\{1,2\}$ and $x \in K$. For all $R \in \mathcal{R}_{s r}^{N}$ and all $E \in \mathcal{E}$ such that $x \in \bigcup_{i \in N} \tilde{E}_{i}$, $\varphi^{r c d(x, \tilde{E})}(E)=\varphi^{r d(1)}(E)$. For all $R \in \mathcal{R}_{s r}^{N}$ and all $E \in \mathcal{E}$ such that $x \notin \bigcup_{i \in N} \tilde{E}_{i}$, $\varphi^{r c d(x, \tilde{E})}(E)=\varphi^{r d(2)}(E)$. Then, $\varphi^{r c d(x, \tilde{E})}$ is efficient, individually rational, and myopic transfer-proof.

Many other restricted conditional dictatorship rules that are myopic transfer-proof and are similar to those in Example 11 can be constructed. For instance, one can condition the choice of the restricted dictator differently on the set of collectively owned objects, e.g., by $K^{\prime} \cap \bigcup_{i \in N} \tilde{E}_{i} \neq \emptyset$ instead of $x \in \bigcup_{i \in N} \tilde{E}_{i}$. Hence, the class of rules that are efficient, individually rational, and myopic transfer-proof for two agents with separable and responsive preferences is very large.

The next example demonstrates that for more than two agents, a restricted serial dictatorship rule may be manipulable by transfers. This result holds for any subdomain of $\mathcal{R}_{u}$ that includes the domain of additive preferences $\mathcal{R}_{a}$, in particular, for $\mathcal{R}_{a}, \mathcal{R}_{s r}, \mathcal{R}_{s}$, and $\mathcal{R}_{u}$.

Example 12. Let $N=\{1,2,3\}, E=\left(E_{1}, E_{2}, E_{3}\right)$ be such that $E_{1}=a, E_{2}=b c, E_{3}=d e$, and $\left(R_{1}, R_{2}, R_{3}\right) \in \mathcal{R}_{a}^{N}$ be the same as in Example 7 .

If agent 1 is the restricted dictator, then the restricted serial dictatorship rule picks $(c d, a e, b)$. However, if agent 3 transfers object $e$ to agent 2, for the resulting exchange market the restricted serial dictatorship rule picks $(b c e, d, a)$. Hence, agent 3 consumes $a$, which she prefers to $b$, in violation of myopic transfer-proofness. Since agent 2 receives $d$, which she prefers to ae, we also have a contradiction to weak transfer-proofness. 


\section{References}

Barberà S, Sonnenschein H, Zhou L (1991) Voting by committees. Econometrica 59: 595-609

Ehlers L, Klaus B (2003) Coalitional strategy-proof and resource-monotonic solutions for multiple assignment problems. Soc Choice and Welfare 21: 265-280

Fiestras-Janeiro G, Klijn F, Sánchez E (2004) Manipulation of optimal matchings via predonation of endowment. Math Soc Sci 47: 295-312

Klaus B, Peters H, Storcken T (1997) Reallocation of an infinitely divisible good. Econ Theory 10: 305-333

Klaus B, Miyagawa E (2002) Strategy-proofness, solidarity, and consistency for multiple assignment problems. Int J Game Theory 30: 421-435

Leontief W (1936) Note on the pure theory of capital transfers, in: Explorations in economics: notes and essays contributed in honor of FW Taussig, McGraw Hill

Ma J (1994) Strategy-proofness and the strict core in a market with indivisibilities. Int J Game Theory 23: 75-83

Pápai, S (2003) Strategy-proof exchange of indivisible goods. J Math Econ 38: 931-959

Pápai, S (2004) Exchange in a general market with indivisible goods. J Econ Theory, forthcoming

Postlewaite A (1979) Manipulation via endowments. Rev Econ Stud 46: 255-262

Roth A (1982) Incentive compatibility in a market with indivisibilities. Econ Letters 9: $127-132$

Roth A (1985) The college admissions problem is not equivalent to the marriage problem. J Econ Theory 36: 277-288

Sertel MR, Özkal-Sanver I (2002) Manipulability of the men- (women-) optimal matching rule via endowments. Math Soc Sci 44: 65-83

Shapley L, Scarf H (1974) On cores and indivisibility. J Math Econ 1: 23-28

Sönmez T (1999) Strategy-proofness and essentially single-valued cores. Econometrica 67: $677-689$

Thomson W (1987) Monotonic allocation mechanisms. Mimeo, University of Rochester 